\title{
Rules for Amalgamating the Reliability of Elements of Powertrain Systems at Maintenance
}

\author{
S. V. Belodedenko, V. I. Hanush and O. M. Hrechanyi \\ The Department of Metallurgical Production Machines and Units, National Metallurgical Academy of Ukraine, Dnipro 49600, \\ Ukraine
}

\begin{abstract}
The amalgamation procedure is actual when using structural methods of reliability. This may apply as the case of the action of a complex of damaging processes on a structural element and the case of the action of a certain damaging process on a system of elements. Classical methods of reliability are poorly adapted for powertrain mechanical systems with a series structural scheme of elements subjected to the gradual influence of several degradation processes. The problem of amalgamation of individual indicators is exacerbated at the stage of operation when diagnosing the technical state of the mechanical system. The application of the classical rule of amalgamation by multiplying the probabilities of survival leads to the effect of over-maintenance. New rules of amalgamation have been developed, which deprive the assessment of the reliability of excessive conservatism. The complex index of the technical condition is offered - the resource safety index (RSI). Its use determines the remaining lifetime. The algorithm for searching the RSI contains an assessment of the criticality of the failure. The search of RSI under the influence of the complex of damaging processes on the element of the powertrain system is demonstrated. The efficiency of the RSI method is shown by the example of the reliability assessment of aircraft bolts. Application of the RSI method increases the guaranteed lifetime by 4-10 times compared with traditional methods.
\end{abstract}

Key words: Reliability indices, powertrain units, maintenance, resource, lifetime, risk.

\section{Amalgamating of the Reliability} Indicators: What Is Meant, When and Where It Is Needed

In terms of assessing reliability, there are two approaches. The combined approach is that the entire mechanical system as a whole is tested or observed. The mean time between failures $t_{\Sigma}$ is used as the primary information, after which a statistical sample of $n$ members is formed. It is processed by appropriate methods, after which the type of the reliability function $P_{c l}(t)$ is established (Fig. 1). Such an approach is sometimes called the classical reliability methods [1]. It is dominated by mathematical-statistical methods, invariant to the type of failure. They are designed, first of all, for electronic systems with a large number of elements operating in parallel, subject to sudden

Corresponding author: Sergei V. Belodedenko, doctor of eng. sciences, professor, head of chair, research fields: reliability and safety of machines, fatigue of metals. failures. For the power mechanical systems, representing, predominantly, a series-connected structure, undergoing gradual effects of several degradation processes, these methods are poorly adaptable.

The main problem of the combined approach is related to the limited sample of the number of facts of failure $n$, which does not allow to correctly determine the form of the reliability function. Reliability tests of full-scale mechanical systems are expensive, therefore it is advantageous to investigate reliability during operation. At a considerable interval of system operate, fails only its non-critical elements, although interest is information that contributes to insurance against significant losses.

Such information in sufficient quantities can be obtained at a lower level by testing small-sized structural elements. In relation to this, algorithms have been developed for obtaining of the lifetime distribution functions (LDF) of certain critical elements of a mechanical system. This is an individual approach to 


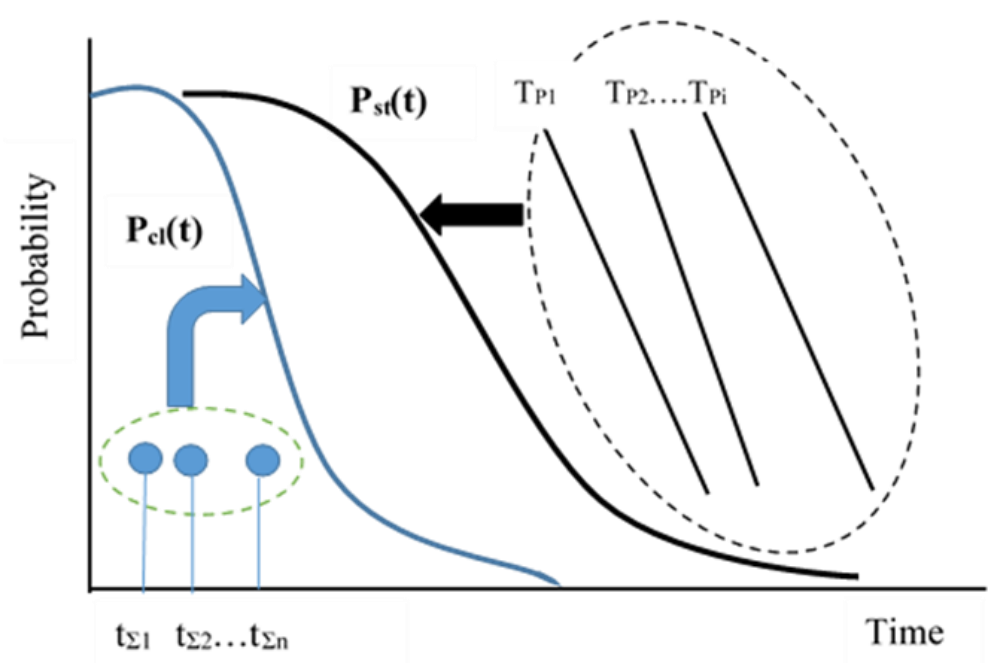

Fig. 1 Two approaches to assessing the reliability of systems.

assessing reliability, which is also called structural reliability methods [1]. Based on the amalgamation, according to the relevant rules of the $T_{R}$ function family, the reliability function $P_{s t}(t)$ is obtained (Fig. 1). In the individual approach, probabilistic-physical methods are used, taking into account the nature of the degradation processes (the failure physics) and the mutual influence of elements $[2,3]$.

In the modern theory of maintenance, the boundary condition is determined by the moment of transition of the system to the corresponding phase of the technical condition, the number of which quantity tends to increase [3]. The same trend is observed in terms of the number of damaging processes affecting the system.

Mathematical-statistical methods of the classical approach should be used in maintenance strategies with reliability centered maintenance. This applies to facilities of mass production, a significant number of homogeneous copies of which are operated in the same conditions. For unique production facilities, it is advisable to use a risk-based strategy. An individual approach together with probabilistic-physical methods is adequate to it. However, one should not oppose the two approaches one should skillfully use the advantages of both methods [1].

When optimizing the periodicity of inspections by the criterion of the minimum cost of operation, one should have a reliability function $P(t)$. In traditional optimization algorithms of Dhillon, Pham, Christer it is obtained by classical methods [4-6]. In this case, the function $P(t)$ is evaluated a posteriori according to the facts of system failures. The latter have a different scale for consequences, as a result of which the reliability function gets a "blurred" character. This leads to a reduction periods between inspections and over-maintenance effect.

The development of technical diagnostic tools made it possible to monitor the most dangerous damage of the fatigue type. This led to the emergence of algorithms Reliability Based Optimization (RBO) [7-9]. In them, the reliability function is estimated based on the probability of defect detection. Their critical sizes and growth intensity are predicted by probabilistic-physical methods. Namely, both fracture mechanics models and fatigue resistance models ( $\mathrm{S}-\mathrm{N}$ models) are used [10]. The effectiveness of RBO-algorithms is high if the type of cracks is known. In fact, the "weak link" principle is used here. In case of multifocal damage, the obtained reliability indices need anyway amalgamated.

The amalgamation procedure is actual when using structural reliability methods. This may be in the action of a complex of damaging processes $D_{l . . k}$ on the 
structural element $E$, and in the action of a certain damaging process $D_{1}$ on the system of elements $E_{1 \cdot * i}$.

The concept of acceptable risk, on which the theory of industrial safety is based, provides for a staged reassignment of service lifetimes (updating). The question of the form of the LDF $T_{P}$ and the inverse of the reliability function $P(t)$ is the key in this situation. If, in the classical approach, the reliability function is considered to be the initial index of the system (which is mostly of a posteriori nature), then in the structural approach for the initial indicator it is necessary to accept the LDF of the elements having a priori nature.

The importance of amalgamating algorithms in maintenance is due to the fact that the planning of recovery operations takes place, at least, at the machine level. It is inappropriate to appoint inspections for only one element, since at that time the entire system will not be available. The amalgamation of indicators is carried out according to some rules. This paper is devoted developing the new rules of amalgamation, which accord to the technical condition maintenance strategy.

The problem of amalgamation of individual indicators is exacerbated at the stage of operation when diagnosing the technical state of the mechanical system. After all, in addition to the considered indicators of the elements, it is necessary to take into account the influence of the complex of degradation processes on them. In addition, thanks to the development of technical diagnostic systems, the number of technical indicators that are heterogeneous has increased. They are also subject to integration in the process of assessing the state of the object for the adoption of one of the three decisions: (1) Prolongation of its operation with standard parameters, or with their limitations; (2) repairs and upgrades with subsequent use in order; (3) decommissioning.

In order to assess the technical condition of powertrain units, it is necessary to use complex (integrated) indicators. The probability of survival $P$ (PS) is used as a complex diagnostic indicator for facilities mass production. In the classical formulation, PS describes the relative number of failures. On the one hand, the prediction algorithms for this indicator should not be too sensitive to the growth of the number of technical system elements. The upward trend in the number of calculated and diagnosable elements may lead to an unjustifiably low predicted level of the PS of the entire system. As a result, the cost of the object increases. On the other hand, PS should respond to the operating time if it acts as a diagnostic parameter. Both conditions contradict each other. This circumstance motivates the search for better indicators.

For a particular element of the mechanical system, the PS ceases to characterize the relative number of failures. Therefore, it requires indicators that are filled with physical content. In this aspect, it is promising to obtain a diagnostic indicator related to the residual operating time (residual, remain resource).

\section{Rules of Amalgamating Individual Reliability Indices for the Reliability Indicator of the Whole Powertrain Unit}

Powertrain units are used in drives of machines for the transfer of efforts, energy and its transformation. These, as a rule, include gearboxes, motors, engines transmissions, pumps. From the standpoint of reliability, the powertrain, most often, is modeled by a series of connecting elements, the failure of which leads to the loss of the available unit.

The development of technical diagnostics makes it possible to observe the effects of the complex of damaging processes. This ultimately increases reliability. Simple technical systems are presented as complex. This is accompanied by modernization of techniques for predicting the technical condition of objects. In such circumstances, amalgamating the indicators of individual elements of the system into a common, generalized system indicator becomes an important process.

The most commonly used method of amalgamating the individual probabilities of the survival (PS) $P_{i}$ of 
individual elements of the system into its common PS $P_{\Sigma}$ is the rule of multiplication:

$$
P_{\Sigma I}=\prod_{i=1}^{i=n} P_{i}
$$

In this case $n$-number quantity of system elements and degradation processes acting on them. The rule of multiplication of the PS corresponds to the rule of adding individual failures ratio: $\lambda_{\Sigma}=\Sigma \lambda_{i}$. Hence for the exponential law of reliability we obtain:

$$
P_{\Sigma I}=\exp \left(-\lambda_{\Sigma} t\right)
$$

The widely-known disadvantage of this rule is an excessive drop in the value of $P_{\Sigma}$ with an increase in the number of elements of the system (line PI, Fig. 3). It is established that calculating the reliability of systems based on the exponential distribution (lambda method) leads to a huge methodological error-to underestimate the estimation of the mean time to failure of the system in $\sqrt{ } n$ times, where $n$ is the number of elements in the consecutive system.

In this regard, the multiplication rule is used to estimate the lower bound of reliability in the first approximation [11]:

$$
\prod P_{i} \leq P_{\Sigma} \leq P_{i \min }
$$

The tighter boundaries based on the probability of compatible events taking into account the mutual correlation of failures were later developed [12].

In the design stage significant decrease the reliability of the system relative to the reliability of its elements provocative the rise of cost mechanical systems. This happens either through the growth of machines weight, or through the use of more high-quality and expensive materials and technologies. The high cost of the system contributes to the fact that the optimal period of its maintenance is reduced.

Practical recommendations for using the multiplication rule are established. (1) If the coefficient of variation of the lifetime elements of the system does not exceed $40 \%$, then you can consider only 5 elements to find the value of $P_{\Sigma}$; (2) It is also possible not to take into account PS elements whose durability is more than 5 times higher than the durability of the weak link [13].

With regard to the reliability of gearboxes, the upgraded Eq. (1) is proposed:

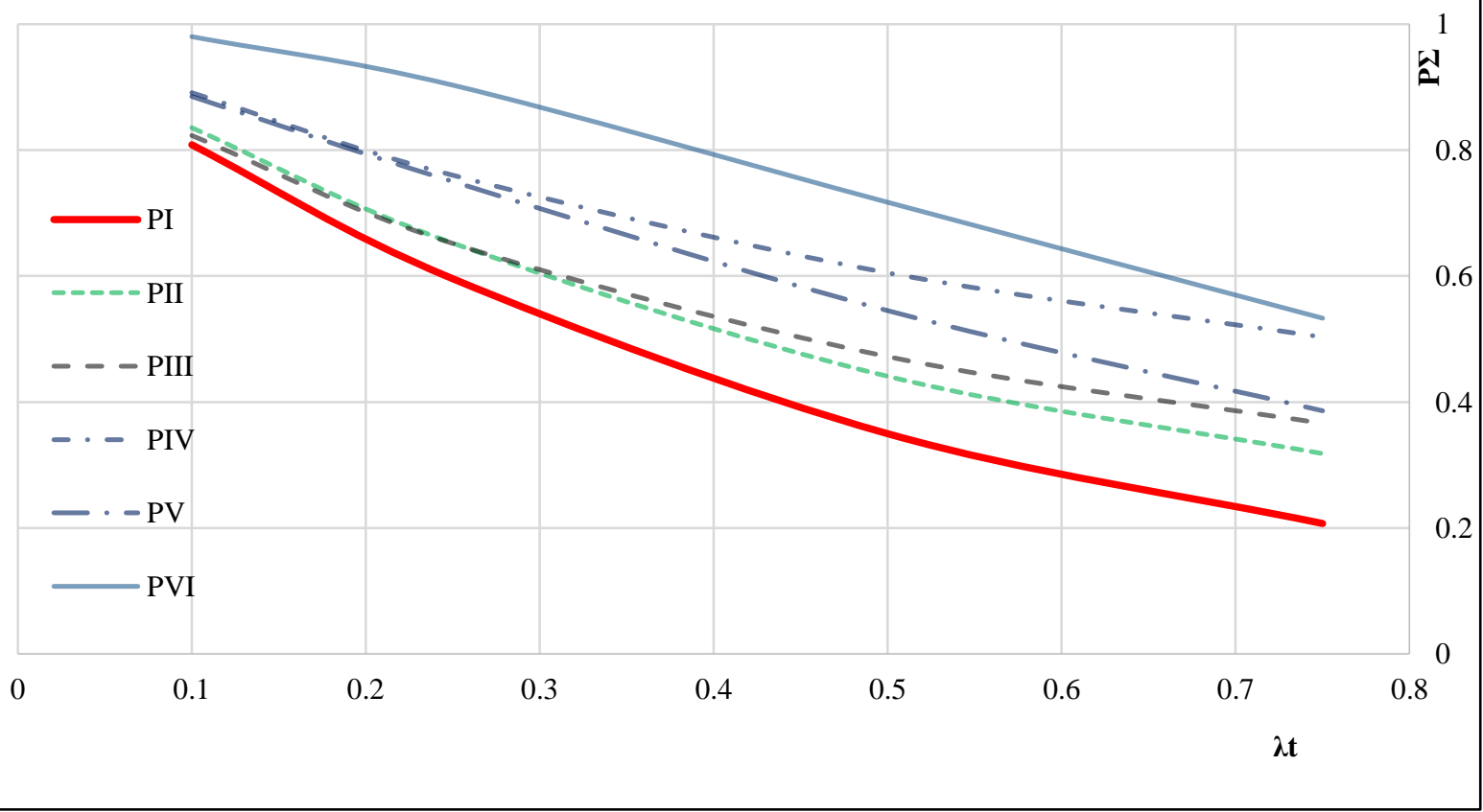

Fig. 2 The change of the reliability in the relative operation time $\lambda t$ of the system $P \Sigma$, which consists of 10 elements (their failure ratio: $\lambda_{1}=\lambda_{2}=\lambda_{3}=0.1$ month $^{-1}, \lambda_{4}=\lambda_{5}=\lambda_{6}=0.2$ month $^{-1}, \lambda_{7}=\lambda_{8}=\lambda_{9}=\lambda_{10}=0.3$ month $\left.^{-1}\right)$. It is obtained by the rules $(1)$ (PI), (2) (PII), (3) (PIII), (4) (PIV, $\left.z_{n}=0.5\right)$, (5) (PV, [1-U] = 0.5), (8) (PVI). 


$$
P_{\Sigma I I}=0,14+0,86 \prod P_{i}
$$

For a definitive determination of the reliability of series systems, the "formula of chain" has become widespread:

$$
P_{\Sigma I I I}=\left[\sum \frac{1}{P_{i}}-(n-1)\right]^{-1}
$$

The results obtained in both formulas are not very different from the dependence of $P_{\Sigma I}(\lambda t)$ (lines PII, PIII, Fig. 2).

The influence of the dependence between elements of the system is taken into account through the coefficient $z_{n}$. In this case, the following dependence [14] applies:

$$
P_{\Sigma I V}=\prod P_{i}+z_{n}\left(\min P_{i}-\prod P_{i}\right)
$$

The coefficient $z_{n}$ increases with the increase in the number of elements of the system $n$, and decreases with the growth of the statistical reserve. Due to this, the decrease in the level of $P_{\Sigma}$ occurs less intensively. The given method flexibly regulates system's reliability (line PIV, Fig. 2), but requires additional research coefficient $z_{n}$.

The multiplication rule gives a pessimistic estimate of predicted reliability, which, most often, is not supported by experiments. A similar phenomenon is known in the methodology of risk analysis. Its main tool is the relationship between cumulative frequency of an accident and its severity of consequences (F-N curves, Farmer's curve), has a form of graduate function. Its degree is the risk aversion factor. A situation arises when a mechanical structural does not perceive risk at the expected level, demonstrating a more optimistic scenario. In this case, the dependence is called a risk aversion curve [15]. This fact gives hope that the use of risk analysis tools will solve the problem of amalgamating the individual ability indicators of individual elements of the system into its common indicator. In its form, the formula used to determine the reliability of systems with structural redundancy is well suited for delaying the fall of the value of $P_{\Sigma I}$ :

$$
P_{\Sigma V}=\left[1+\sum k_{f} \frac{1-P_{i}}{P_{i}}\right] \prod P_{i}
$$

Here the redundancy is estimated through the coefficient $k_{f}<1$. For a simple system of $k_{f}=0$ and the large risk of a failure. With full redundancy of $k_{f}=1$ and the risk of failure (consequences of failure), practically no. Unfortunately, $k_{f}$ values are obtained by testing the entire system. To get rid of this, you can use the coefficients of significance (criticality) of failures that exist in the risk analysis: $u_{i}=1-k_{f i}$. The greater the critically of failure in its consequences, the smaller is the addition to increasing the system's minimum reliability. The fraction in Eq. (5) is a dimensionless risk index $\rho_{i}$ [15]. In essence, this is a odds ratio. With deterministic calculations for $P=0.5$, the risk becomes complete, that is, $\rho=1$. In this case Eq. (5) transforms into:

$$
P_{\Sigma V}=\left[1+\Sigma\left(1-u_{i}\right) \rho_{i}\right] \prod P_{i}
$$

With this dependency, you can effectively influence the amalgamation process. But it is not effective at extreme critical values, i.e. for $u_{i}=0$ and for $u_{i}=1$.

From one of the modifications to the Lindley distribution [16, 17] follows a new rule of amalgamation, which in structure is similar to the structure of Eq. (5). One-dimensional form of Lindley distribution has cdf:

$$
F(t)=1-\frac{\theta+1+\theta t}{\theta+1} \exp (-\theta t)
$$

The scale parameter $\theta$ can be expressed through the failure rate $\lambda: \theta=\lambda /(1+\lambda)$. Then Eq. (6) can be represented as:

$$
\begin{gathered}
F(t)=\theta[1-\exp (-\lambda t)]+ \\
(1-\theta)[1-(1+\lambda t) \exp (-\lambda t)]
\end{gathered}
$$

Hence, if by the addition rule find the total failure rate for the $\lambda_{\Sigma}$ system, its reliability will be found as:

$$
P_{\Sigma V I}=\left(1+\lambda_{\Sigma} t\right) \exp \left(-\lambda_{\Sigma} t\right)
$$


Marking $P_{E X P}=P_{I}$, these formulas are transformed into $P_{\Sigma V I}=P_{L N D}=\left(1+\lambda_{\Sigma} t\right) P_{E X P}$.

From Fig. 2, it is seen that the classic rule of multiplication is the most conservative estimate of the system's reliability. In the opinion of the authors, such a rule should be used at the design stage for mechanical systems consisting of elements of relatively low reliability. To predict the reliability of responsible systems with high-reliability elements it is worth using qualitatively other models, which ensure the principle $P_{\Sigma} \rightarrow P_{i k}$. Moreover, it is advisable to do it at the stage of operation, when some reliability indicators are already known.

As a result of valid studies, it was found that the rules for amalgamating $P_{V}, P_{V I}$ correspond to the principle $P_{\Sigma} \rightarrow P_{i k m i n}$. However, they are sensitive enough to increase the number of system elements. With their increase it is difficult to achieve the desired principle. To solve this problem, one must understand what the cause of such a phenomenon is.

The rule of multiplication of the PS is fair for independent events. If there are deviations from this rule, then it is assumed that these are the consequences of the mutual influence of the elements of the system [14]. This effect is manifested during operation. When forecasting the system's reliability, there is the amalgamation effect that involves a significant reduction of the magnitude $P_{\Sigma}$ relative to the $P_{i k}$ values. The amalgamation effect is formalized, as $P_{\Sigma} \ll P_{i k m i n}$. It is the outcome of uncertainty. Failure of the system occurs under the influence of the dominant damaging process for the most vulnerable element. Combating the effect of the amalgamation is the procedure for identifying (updating) the model of the operation process. Its result is the simplification of a complex technical system to the simple, whose robustness is valued on the principle of weak link. Thus the principle $P_{\Sigma} \rightarrow P_{i k m i n}$ is realized.

This corresponds to the rules of combining risk indicators, one of which is the resource (lifetime) safety index (RSI) [15].

\section{The Resource (Lifetime) Safety Index}

When diagnosing a safe state of the system is evaluated by comparing the indicators of the damaging (degradation) process $y$, acting on the system, and indicators of system resistance to process $Y$. In the general case, performance indicators $Y$ and $y$ with sufficient informativity can serve as indicators. Complex indicators, unlike simple ones, are more informative, but they are more difficult to control. Simple indicators are effective for simple technical systems by the type of "weak link".

In order to determine the probability of survival operation during sudden failures, the model of "load-strength" comparison is usually used. Reliability is defined as the probability of exceeding the strength over the load. The "load-strength" model has been worked out in detail for various combinations of statistical distributions and is a classic approach. As a result this model is also used for failures of a gradual type. But for fatigue failures the conditionally selected limit value of the load can exceed the minimum strength level in many times not reflecting on the actual reliability. But according to the existing model, there should be a failure. To overcome this contradiction several ways not proved to be effective are offered.

The comparative model "operation (operating time)-resource (lifetime)", which distinguishes the resource-based approach, is a comprehensive solution to the problem of gradual failures. It works fine at the operation stage, when the diagnosis of the residual resource is carried out by controlling the natural parameter, which is the operating time $t$. The ratio of maximum operation life $t$ at the time of control to the minimum resource $T_{P}$ (which is determined in the statistical aspect by its own distribution functions) forms a guaranteed safe factor to operation life $n_{T P}$. The logarithm of its current value in the form of the safety index decreases linearly with the operating time. When the RSI reaches zero value it indicates that the object is used with the unacceptable risk. 
The $n_{T P}$ value is inverse to the probabilistic accumulated damage in the resource interpretation $a_{P}$. Then for an individual safety index RSI is fair:

$$
\beta_{P i k}=\lg \frac{T_{P}}{t}=\lg n_{T P}=\lg a_{P}^{-1}=\beta_{P i k 0}-\lg t
$$

The similar use of the risk indicator for sudden type failures is not possible according to the classical model. In general, the model "load-strength" is not adapted for the current control of the technical condition. Therefore, the purpose of the available researches is to use the resource model for sudden failures and develop the algorithm for the safety indexes determination. Harmonization of the technical condition estimation methods as the result gives an opportunity to increase the service reliability.

Dimensionless risk index of the system is determined by the probability of the failure $Q_{i k}$ and its criticality $u_{i k}$ as follows [15]:

$$
\rho_{\Sigma}=\sum Q_{i k} u_{i k}
$$

Taking into account that the deterministic damage $a$ $=t / T_{0}=\lambda t$, the exponential distribution law is transformed as $P_{E X P}=\exp (-a)$. In operational safety strategies, first of all, high-risk elements are considered, for which the deterministic damage, as a rule, does not exceed 0.25 . Then, with an error of not more than 5\%, you can take $P_{E X P}=1-a$. It follows that $a=Q$. Combining Eqs. (9) and (10) gets the RSI for systems:

$$
\beta_{\Sigma P}=\lg \left(\sum \frac{u_{i k}}{10^{\beta_{P i k}}}\right)^{-1}
$$

Guided by the same considerations, we can find the rule for amalgamating the safety indexes for the reliability functions described by the Lindley law $P_{L N D}$ $=(1+a) P_{E X P}$. Then they are represented in the form $P_{L N D}=(1+a)(1-a)=1-a^{2}$. From this it follows that $a^{2}=Q$. Then, analogously to Eq. (11), we obtain:

$$
\beta_{\Sigma P L N D}=\lg \left(\sum \frac{u_{i k}}{10^{2 \beta_{P i k}}}\right)^{-1}
$$

\section{Search of the General Safety Index for a Complex of Damaging Processes}

The effectiveness of the RSI method was demonstrated by the example of high-strength M18 bolts for connecting aircraft structural, including chassis wheels (Fig. 3). The detail is a responsible element. For dangerous places bolt where fractures occurs (1, 2, 3, 4, Fig. 3) obtained the fatigue models (Table 1). Fractures in places 2 and 3 are typical for imperfect bolts.

The first model is the lifetime general equation (LGE) for lifetime (durability) $N$ in the form:

$$
\lg N=b_{0}+m \lg \Delta F+b_{r} R_{\sigma}
$$

where $b_{0}, m, b_{r}-$ coefficients of the model;

$\Delta F$ is the double amplitude (swing) of the force acting on the bolt in $\mathrm{kN}$;

$R_{\sigma}-$ stress ratio.

The second model is the equation for the dispersion of durability (EDD) in the form of:

$$
S_{\lg N}=B+k_{L}\left(\lg N-\lg N_{A}\right)
$$

where $S_{l g N}-\mathrm{SD}$ of the number of cycles to the boundary state;

$B, k_{L}, \lg N_{A}$-coefficients of the model.

For dangerous places bolt where fractures occurs $(1,2,3,4$, Fig. 3$)$ obtained the parameters fatigue models (Table 1).

The task was to find the cyclic lifetime of $N_{P}$, which guarantees the safety of PS $P=0.98$. For this purpose, according to Eq. (9), the value $\beta_{\Sigma P 0}=\lg N_{P}$ is determined (Fig. 3). The calculation is performed for a fixed tightening forces $F$ generated in the body of the bolt tension 0.3 and 0.6 of yield strength. Changing the value of external loading $\Delta F$ alters stress ratio $R_{\sigma}$. Therefore, the use of LGE is convenient.

On the basis of the developed algorithm, the diagrams of the initial RSI $\beta_{\Sigma P 0}$ were obtained (Fig. 3). Since during construction, the external load is considered as a variative, this diagram is, in essence, a fatigue curve for the random load at the PS $=0.98$. 


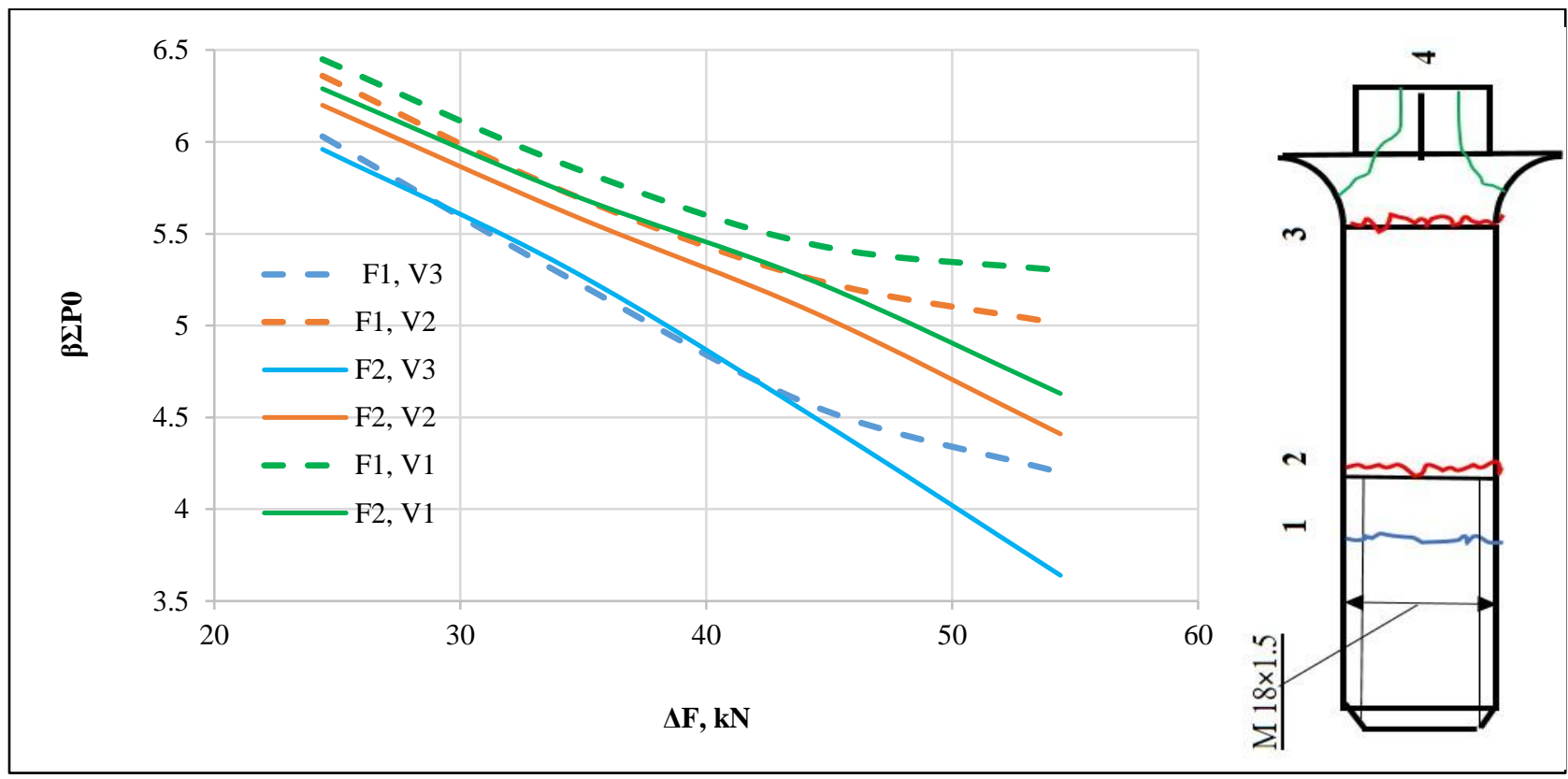

Fig. 3 The initial safety indices $\beta_{\Sigma P 0}$ of the bolt M18 with the tightening forces $F=75.6 \mathrm{kN}(\mathrm{F} 1), F=151.2 \mathrm{kN}(\mathrm{F} 2)$ and the coefficients of the variation of the double amplitude of the load $\Delta F V_{F}=0.05(\mathrm{~V} 1), 0.1(\mathrm{~V} 2), 0.2(\mathrm{~V} 3)$.

Table 1 Parameters of fatigue models for dangerous bolt places and their criticality (LGE is obtained for double the amplitude of the forces $\Delta F(\mathrm{KN}))$.

\begin{tabular}{llllllll}
\hline \multirow{2}{*}{ Dangerous place } & \multicolumn{4}{c}{ LGE } & \multicolumn{2}{c}{ EDD } \\
\cline { 2 - 8 } & $b_{0}$ & $m$ & $b_{r}$ & $B$ & $k_{L}$ & $\lg N_{A}$ & $u_{i k}$ \\
\hline Thread, 1 & 30.7 & -13.2 & -2.5 & 0.16 & 0.16 & 5.0 & 0.66 \\
Head surface, 4 & 14.5 & -4.5 & -2.5 & 0.16 & 0.16 & 5.0 & 0.11 \\
Fillets, 2, 3 & 21.5 & -8.6 & -2.5 & 0.30 & 0.24 & 5.6 & 0.20 \\
\hline
\end{tabular}

Studies have shown that the factor of variation in external loading of $V_{F}$ has a more significant effect on the guaranteed lifetime than the tightening force $F$. From the diagrams is showed that RSI method allows 4-10 times longer use of the details than with the forecast by traditional means.

Considering the defined guaranteed lifetime of the object under of the system of damaging processes, we can consider the index of criticality $u_{i k}$ as a powerful instrument for the regulation of amalgamated reliability. Such a conclusion follows from the fact that the guaranteed lifetime increases 4-5 times in the transition from the situation $u_{i k}=1$ to the algorithm with the actual calculated $u_{i k}<1$. That is, in the first situation, the amalgamated RSI is significantly smaller than the mean $\beta_{m P}$ between the individual indexes: $\beta_{\Sigma P}$ $<\beta_{m P}$. In the second situation, the principle $\beta_{\Sigma P} \rightarrow \beta_{m P}$, which can be considered analogous to the above principle $P_{\Sigma} \rightarrow P_{i k m i n}$, is formalized.

\section{Conclusions}

The structure of amalgamating formulas, which exclude excessive conservatism when calculating the reliability of the system, is found. New rules for amalgamating based on the risk indicator and on the basis of Lindley's distribution are obtained.

The algorithm for search of the safety index for systems is proved, which ensures the implementation of the principle $\beta_{\Sigma P} \rightarrow \beta_{m P}$, which can be considered analogous to the principle $P_{\Sigma} \rightarrow P_{i k m i n}$. The rule for amalgamating individual indices based on the distribution of Lindley $\beta_{\Sigma P L N D}$ is proposed. It is recommended to use it for a large number of (more than 10) critical elements of the system and for 
multi-site damage. With less number of them it is suggested to use a more usual form of the RSI $\beta_{\Sigma P \text {. It }}$ well meets the situation of several (4-7) degradation processes on the element of the technical system.

An explanation of the low reliability of the system, which is determined by the rule of multiplication of the PS, is found. Usually it is associated with the factor of mutual influence of elements. In the method of the safety index, the situation $P_{\Sigma} \ll P_{i k m i n}$ is explained by the uncertainty of models. Its influence is offset by the identification of models, among which the criticality of failure $u_{i k}$ is important.

The algorithm for constructing the diagram "RSI of the system $\beta_{\Sigma P}$ - load parameter (in this case $-\Delta F$ )" is a alternative to the procedure of summation of the damage. The latter is relevant at the early stages of design, when the uncertainty of the loading to be chosen the spectrum with a wide variation. At the stage of operation, when the loading process is monitored, its variation is substantially reduced. It creates the opportunity not to sum up the damage directly to control the exhaustion of the resource or the remaining lifetime.

\section{References}

[1] Fischer, K., Asmolovkiy, N., Custer, R., Schubert, M., Keil, D., Braun, B., and Faber, M. H. 2016. "New Approaches for Reliability Assessment of Mechanical Systems and Parts." Submitted to ECSSMET. www.researchgate.net/publication/308898708 [Accessed 01/11/2016]. 15p.

[2] Algin, V. B., and Kim, H.-E. 2006. "Reliability and Lifetime of Mechanical Units in Operation and Test." Key Engineering Materials 326-8:549-52.

[3] Belodedenko, S. V., and Ibragimov, M. S. 2017. "Models for Optimization the Preventive Maintenance Schedules of Mechanical Systems in Metallurgy." Metallurgical and Mining Industry 2: 26-37.

[4] Dhillon, B. S. 2008. Mining Equipment Reliability, Maintainability and Safety. London: Springer.

[5] Wang, H., and Pham, H. 2006. Reliability and Optimal
Maintenance. Springer: Verlag London Ltd.

[6] Christer, A. H., and Waller, W. M. 1984. "Delay Time Models of Industrial Inspection Maintenance Problems." Journal of the Operational Research Society 35: 401-6.

[7] Frangopol, D. M. 2011. "Life-cycle Performance, Management, and Optimization of Structural Systems under Uncertainty: Accomplishments and Challenges." Structure and Infrastructure Engineering 7 (6): 389-412.

[8] Zou, G., Banisoleiman. K., and González, A. 2017. "Reliability-based Inspection Planning in View of Both Crack Initiation and Propagation." In Safety and Reliability. Theory and Applications, edited by Cepin, M., and Bris, R. CRC Press, 540.

[9] Valdebenito, M. A., and Schueller, G. I. 2010. "Design of Maintenance Schedules for Fatigue-prone Metallic Components Using Reliability-based Optimization." Comput. Meth. Appl. Mech. \& Eng. 2: 36.

[10] Van Danga, T., Anh Maia, Q., Moratoa, P. G., and Rig, P. 2018. "Updating the Failure Probability of Miter Gates Based on Observation of Water Levels." In Proceedings of the 19th International Colloquium on Mechanical Fatigue of Metals, 53-5. http://icmfm19.com/wp-content/uploads/2018/09/BookOf Abstracts_ICMFM-XIX.pdf.

[11] Cornell, C. A. 1967. "Bounds on the Reliability of Structural Systems." Journal of Structural Division, ASCE 93 (ST1): 171-200.

[12] Ditlevsen, O. 1979. "Narrow Reliability Bounds for Structural Systems.” Journal of Structural Mechanics, ASCE 7 (4): 453-72.

[13] Reshetov, D. N., Ivanov, A. S., and Fadeev, V. Z. 1988. Reliability of Machines. Moscow: High sch. (in Russian)

[14] Pereverzev, E. S., and Daniev, Y. F. 1999. Tests and Reliability of Technical Systems. Dnepropetrovsk: NAS, NCA of Ukraine, ITM. (in Russian)

[15] Belodedenko, S. V., and Bilichenko, G. N. 2015. "Quantitative Risk-Analysis Methods and Mechanical Systems Safety." Metallurgical and Mining Industry 12: 272-9.

[16] Espirito Santo, A. P. J., and Mazucheli, J. 2016. "The Composed Zero Truncadet Lindley-Poisson Distribution." Pesqui. Oper. 36.

[17] Mansour, M. M., and Hamed, S. M. 2015. "A New Generalization of Power Lindley Distribution: With Application to Lifetime Data." Journal of Statistics: Advances in Theory and Applications 13: 33-65. 